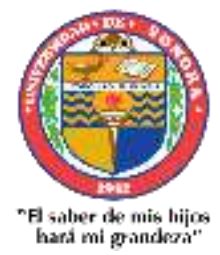

( Enero - junio 2020 )

Año 13.

Núm. 32

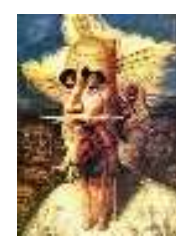

Revista de Investigación

Académica sin Frontera

ISSN: 2007-8870

http://revistainvestigacionacademicasinfrontera.com

Recibido el 24 de octubre de 2019. Dictaminado mediante arbitraje favorablemente 15 de enero de 2020.

\title{
Medición de la satisfacción de los servicios universitarios en estudiantes de Posgrado: estudio en una Universidad Pública.
}

\section{Measurement of the satisfaction of university services in postgraduate students: study in a Public University.}

\author{
Yesenia Roseles Jiménez Silván* \\ Fabiola De Jesús Mapén Franco** \\ Germán Martínez Prats***
}

\section{Resumen}

Se considera que la evaluación de los servicios universitarios es apropiada cuando se integran opiniones e indicadores de satisfacción estudiantil. Aunque se dispone de instrumentos para medir la satisfacción estudiantil a nivel institucional, los reportes generalmente no muestran la confiabilidad de estos, es por ello que se dispuso a realizar la presente investigación tomando como base el cuestionario presentado por Jaqueline (2006), el cual mide la satisfacción de los servicios universitarios desde la perspectiva estudiantil, se buscó adaptarlo y aplicarlo de acuerdo a la investigación presentada. Se aplicó un total de 50 encuestas a estudiantes de los diferentes posgrados que ofrece la División Académica de Ciencias Económico Administrativas (DACEA) una de las 12 divisiones que integran la Universidad Juarez Autonoma de Tabasco ( UJAT). El cuestionario esta integrado por 47 ítems que a su vez se encuentran clasificados en 10 dimensiones diferentes. Dentro de los resultados obtenidos se encuentró que la dimensión con menor grado de satisfacción fue la de servicios informáticos, ya que los alumnos se encuentran algo insatisfechos con la plataforma para reinscribirse y la dimensión que obtuvo mayor grado de satisfacción fue la de calidad docente ya que opinan que el profesor cumple con el perfil para ejercer dicha profesión.

Palabras clave: satisfacción estudiantil, servicios universitarios, Calidad educativa.

\footnotetext{
*Pasante de la Licenciatura en Administración en la División Académica de Ciencias Económico-

Administrativas (DACEA) de la Universidad Juárez Autónoma de Tabasco.

**Dra. En Estudios Organizacionales. Profesora-Investigadora de la Universidad Juárez Autónoma de Tabasco. ORCID: https://orcid.org/0000-0002-8436-4615

*** Dr. en Gobierno y Administración Pública. Profesor-Investigador de la Universidad Juárez Autónoma de Tabasco. ORCID: https://orcid.org/0000-0001-6371-448X
} 


\begin{abstract}
The evaluation of university services is considered appropriate when integrating opinions and indicators of student satisfaction. Although instruments are available to measure student satisfaction at the institutional level, the reports generally do not show their reliability, which is why they prepared to carry out this research based on the questionnaire presented by Jaqueline (2006), which measures Satisfaction of university services from a student perspective, we sought to adapt it and apply it according to the research presented. A total of 50 surveys were applied to students of the different postgraduate courses offered by the Academic Division of Administrative Economic Sciences (DACEA), one of the 12 divisions that make up the Juarez Autonomy university of Tabasco (UJAT). The questionnaire is made up of 47 items that in turn are classified into 10 different dimensions. Among the results obtained, it was found that the dimension with the lowest degree of satisfaction was that of computer services, since the students are somewhat dissatisfied with the platform to re-enroll and the dimension that obtained the highest degree of satisfaction was that of teaching quality since they believe that the teacher complies with the profile to exercise said profession.
\end{abstract}

Keywords: student satisfaction, university services, educational quality.

\title{
Introducción
}

"El alumnado universitario como actor beneficiario de la formación académica constituye un indicador fundamental para el estudio de la calidad de las instituciones. Estudiantes satisfechos con la formación recibida identifica y confirma la calidad de la misma, lo que redunda en una mayor motivación e implicación, pero también en el desarrollo de un sentimiento de pertenencia y deseos de intervenir en los distintos cauces de participación favoreciendo una difusión positiva de la actividad académica y la permanencia en las aulas”. (Sánchez, García, \& Martínez, 2012).

Para Gento \& Vivas (2003) la satisfacción es el resultado de un proceso valorativo. En consecuencia, cuando un individuo manifiesta satisfacción o rechazo hacia algo, está emitiendo un juicio de valor a partir de un proceso de evaluación.

Estudios realizados sostienen que los estudiantes que presentan un alto grado de satisfacción con la universidad se caracterizan por realizar adecuadamente sus metas académicas, poseer elevadas creencias acerca de sus capacidades para lograr un buen rendimiento en las tareas, desarrollar expectativas positivas respecto a la vida universitaria (Medrano \& Pérez, 2010).

Es por ello que la satisfacción estudiantil juega un papel muy importante en las instituciones ya que si es positiva trae beneficios para los alumnos y la institución, pero si es negativa afecta la formación académica y el desempeño de los estudiantes. 
Los servicios universitarios pueden ser explícitos o implícitos. El servicio explícito incluye los niveles de conocimiento del personal, la capacidad docente del personal, la coherencia de la calidad de la enseñanza independientemente del personal, la facilidad para concertar citas con el personal, el nivel de dificultad del contenido de la asignatura y la carga de trabajo (Cadena, Mejías, Vega, \& Vásquez, 2015; Bendermacher, Egbrink, Wolfhagen, \& Dolmans, 2017)

Es decir, todos aquellos servicios que se pueden apreciar por medio de los sentidos. Por ejemplo, si un profesor está realmente capacitado para brindar clases se refleja por la forma en que se desempeña y el dominio que tiene de la asignatura.

El servicio implícito incluye el tratamiento de los alumnos por parte del personal, incluida la amabilidad y la accesibilidad, la preocupación que se muestra si el alumno tiene un problema, el respeto por los sentimientos y opiniones, la disponibilidad del personal, la capacidad y la competencia del personal. (Lomas, MacGregor, \& Hill, 2003).

Es decir, son aquellos servicios que no se observan tan evidentes pero que se deduce que deben proporcionarse a los alumnos universitarios.

La satisfacción de los estudiantes con relación a la educación que están recibiendo es una referencia constante con un valor clave en la calidad (Allen, Bourhis, Burrell, \& Mabry, 2002; Eom, Wen, \& Ashill, 2006; Groenewegen \& Hutten, 1991; Sanjuan, Gómez, Rabell \& Morales, 2011; Zambrano ,2016) Las investigaciones sugieren que el fortalecimiento de la calidad de la institución de educación superior está relacionado con aspectos que influencian la vida estudiantil (Tam, 2002). Se ha encontrado que el contexto del establecimiento educativo puede influenciar en los estados intelectuales, sociales, emocionales y culturales, como efecto de los servicios institucionales.

Sin embargo, sigue siendo un desafío para los administradores y autoridades educativas conocer los factores que pueden aumentar la satisfacción de los estudiantes como aumentar la calidad del servicio. La satisfacción de los estudiantes es el centro de las preocupaciones de los encargados de las instituciones ya que si se brindan los servicios universitarios de manera correcta ayuda al rendimiento académico y a su vez aumenta la calidad del servicio.

Una dificultad en los esfuerzos de aumentar la calidad en los servicios a los estudiantes es el desconocimiento de cuáles son los factores importantes para los estudiantes. En algunas investigaciones se pueden encontrar evaluaciones que pretenden identificar el grado de satisfacción, pero usualmente no tienen en cuenta cuán importante es un factor en particular. Por esta razón, este estudio tiene el propósito de medir el grado de satisfacción de alumnos de 
posgrados, clasificando los servicios en dimensiones para identificar cuál de ellas tiene el mayor grado de satisfacción y cuál el menor, de esta manera será posible definir acciones de mejora en la institución educativa.

\section{Calidad de servicio en el ámbito educativo}

La calidad que fue utilizada en el ámbito empresarial fue trasladada al ámbito educativo, cuando el concepto se aplica a este ámbito es necesario considerar la finalidad, el sentido y funciones de la educación. Considerando al sistema educativo en su conjunto ( Sverdlick, 2012).

Para Senlle \& Gutiérrez (2005), la calidad no consiste en aplicar las normativas internacionales aprobadas por 157 países o incrementar ya sea las horas de clase o los exámenes; la calidad es un cambio profundo en la manera de pensar, de programar, de evaluar y de sentir la educación. El afán eficientista llevó a muchas instituciones a preocuparse por la calidad dejando de lado el asegurar la enseñanza y aprendizaje en los estudiantes. Se puede hablar de calidad en educación, cuando esta es causante del progreso de sus estudiantes en un amplio grado de beneficios intelectuales, morales, emocionales y sociales, teniendo en cuenta su nivel socioeconómico, su medio familiar y su aprendizaje previo. Un sistema educativo eficaz es el que maximiza la capacidad de las instituciones para alcanzar esos resultados (Bustos, Salazar, \& Bermúdez, 2012; Aguerrondo, 2003) enfatizaba la fuerza del concepto de calidad al tratarse de un concepto totalizante, amplio y multidimensional. Por otro lado (Capelleras \& Veciana, 2004) percibieron el creciente interés por la evaluación de la calidad de la enseñanza que no esté exclusivamente centrada en la labor del profesor y elaboraron una síntesis de las investigaciones desarrolladas en este ámbito. Ciertos trabajos se han centrado en la valoración de la experiencia global de los estudiantes en la universidad con relación al conjunto de servicios que ofrece no sólo a nivel docente, sino que también se incluyen los servicios de apoyo al estudio y los servicios generales tales como los relacionados con el desarrollo deportivo, cultural y de convivencia. Las principales conclusiones a las que arribaron en el contexto de la educación superior fueron: no existe unanimidad en cuanto a la conceptualización de la calidad de servicio en el ámbito universitario desde la perspectiva del usuario, tampoco una escala estandarizada para medir esta temática y, por otro lado, los resultados varían puesto que no siempre se mide el mismo concepto. Esto ha obligado 
de alguna manera a que exista una marcada preocupación de los gobiernos por la evaluación de la calidad dentro de los sistemas educativos.

La preocupación por la calidad de la educación que están mostrando las universidades, se ha pronunciado en el nuevo milenio (Capelleras \& Veciana, 2004; Salvador, 2005; Senlle \& Gutiérrez, 2005), relacionan la fórmula de la calidad en la educación con la estructura y gestión de la institución educativa y con la metodología o estrategias para transmitir el conocimiento con el fin de cambiar y desarrollar competencias. Si los servicios no son de calidad, deterioran el clima y los resultados institucionales. (Senlle \& Gutiérrez, 2005) señalaban "que cuando hay desconexión entre los estudios y lo que requiere la sociedad hay falta de calidad." (p.33).

\section{Satisfacción de los servicios universitarios en alumnos de posgrado}

La Universidad Juárez Autónoma de Tabasco, es una universidad pública localizada en el estado de Tabasco, México, además de las diversas licenciaturas que ofrece, también ofrece estudios de posgrados: Doctorado en Estudios Económico Administrativos, Maestría en Administración Educativa, Maestría en Administración, Maestría en Administración y Dirección Estratégica, Maestría en Ciencias en Gestión del Desarrollo Regional, Maestría en Contaduría y Maestría en Gerencia Pública y Gobierno. Actualmente se encuentran inscritos un total de 86 alumnos, se utilizó una muestra de 50 alumnos de manera aleatoria para la aplicación del cuestionario.

Cabe recalcar que los posgrados mencionados están inscritos en el Padrón del Programa Nacional de Posgrados de Calidad (PNPC), el reconocimiento a la calidad de la formación de los programas de posgrado que ofrecen las instituciones de educación superior y los centros de investigación se lleva a cabo mediante rigurosos procesos de evaluación por pares académicos, y se otorga a los programas que muestran haber cumplido los más altos estándares de calidad y pertinencia. (Consejo Nacional de Ciencia y Tecnología [Conacyt], 2019).

Los programas que resultan aprobados en el proceso de la evaluación académica se integran en el Padrón del Programa Nacional de Posgrados de Calidad y está conformado por cuatro niveles:

Competencia internacional. Programas que tienen colaboraciones en el ámbito internacional a través de convenios que incluyen la movilidad de estudiantes y profesores, la codirección de tesis y proyectos de investigación conjuntos.

Consolidados. Programas que tienen reconocimiento nacional por la pertinencia e impacto en la formación de recursos humanos de alto nivel, en la productividad académica y en la colaboración con otros sectores de la sociedad. 
En desarrollo. Programas con una prospección académica positiva sustentada en su plan de mejora y en las metas factibles de alcanzar en el mediano plazo.

De reciente creación. Programas que satisfacen los criterios y estándares básicos del marco de referencia del PNPC. (Conacyt, 2019)

\section{Método}

El estudio se llevó en la División Académica de Ciencias Económico Administrativas (DACEA) una de las 12 divisiones que integran la Universidad Juárez Autónoma de Tabasco (UJAT). Participaron un total de 50 estudiantes de los diferentes posgrados que ofrece la institución, 56\% hombres y $44 \%$ mujeres.

\section{Procedimiento}

Se aplicó el cuestionario para medir la satisfacción estudiantil presentado por (Jacqueline \& Barry, 2006) y se adaptó de acuerdo a la investigación. La validación del instrumento se realizó a través del juicio de expertos con reconocimiento académico y prestigio por sus investigaciones.

La recolección de datos se realizó en las aulas de clases de la División Académica de Ciencias Económico Administrativas (DACEA).

El cuestionario se integra por 53 ítems. Los ítems fueron clasificados en 10 dimensiones las cuales son: ambiente educativo, bienestar estudiantil, calidad administrativa, calidad docente, infraestructura educativa, organización académica, recursos para la investigación, servicios estudiantiles, servicios informáticos y vinculación con la sociedad. Cada ítem fue medido con respecto a su satisfacción, siguiendo el mismo formato usado por (Jacqueline \& Barry, 2006) Para esto se usó una escala de Likert de cinco puntos de la siguiente manera:

1. Muy insatisfecho

2. Un poco insatisfecho

3. Ni Satisfecho, ni insatisfecho

4. Un poco satisfecho

5. Muy satisfecho 


\section{Resultados}

Todos los análisis estadísticos se calcularon usando el programa Excel, y fueron interpretados por dimensión. Los resultados que se obtuvieron fueron los siguientes:

- El total de evaluados fueron 50 estudiantes: $56 \%$ hombres y $44 \%$ mujeres. (Vea Gráfica 1).

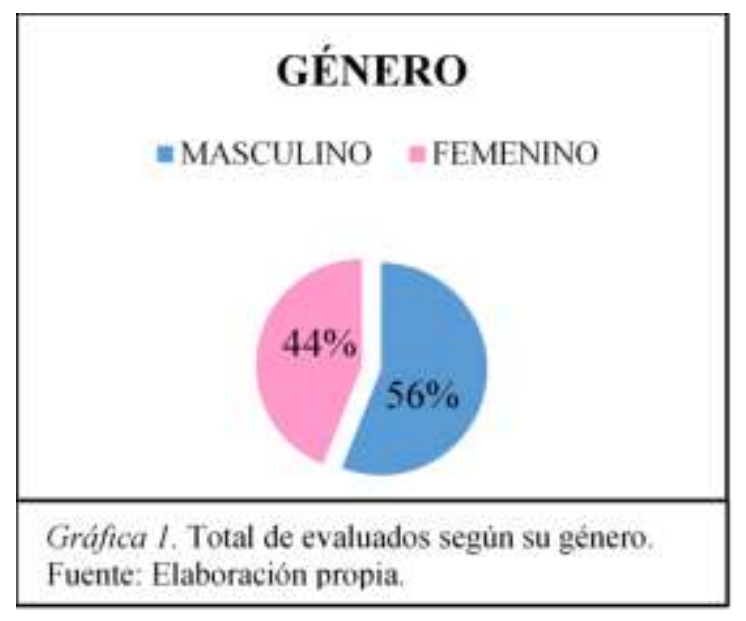

El total de posgrados evaluados fueron 5, Doctorado en Administración Educativa, Doctorado en Estudios Económico Administrativas, Maestría en Administración y Dirección Estratégica, Maestría en Contaduría y Maestría en Gerencia Publica y Gobierno. (Vea Gráfica 2)

Gráfica 2. Total de posgrados evaluados.

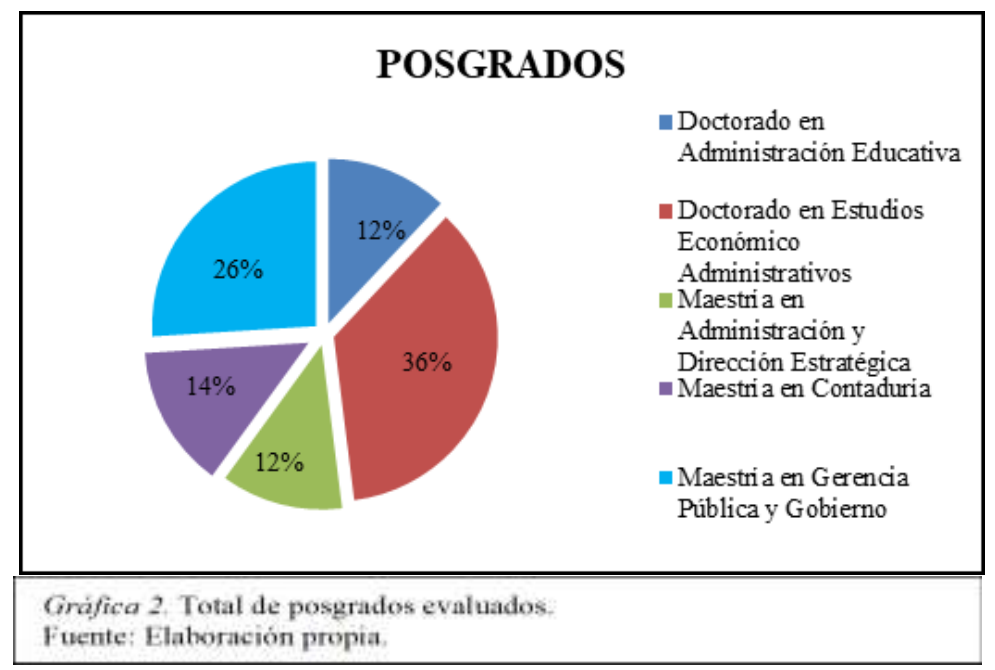


- Primeramente, se determinó el grado de satisfacción de los servicios universitarios mediante el análisis de cada una de las 10 dimensiones.

Se obtuvo que en la categoría de ambiente educativo el $32 \%$ de los estudiantes opina que se encuentran un poco satisfechos con los servicios que abarca dicha categoría y el 5\% opina que se encuentran muy insatisfechos, mencionando algunos servicios que integran dicha dimensión están: Capacidad de respuesta del personal a solicitudes, El nivel de limpieza de los salones de clase, la idoneidad de la cantidad de evaluaciones de las clases, Las clases en general, ellos consideran que la capacidad de respuesta del personal es algo buena al igual que la limpieza y las evaluaciones de las clases. (Vea Gráfica 3)

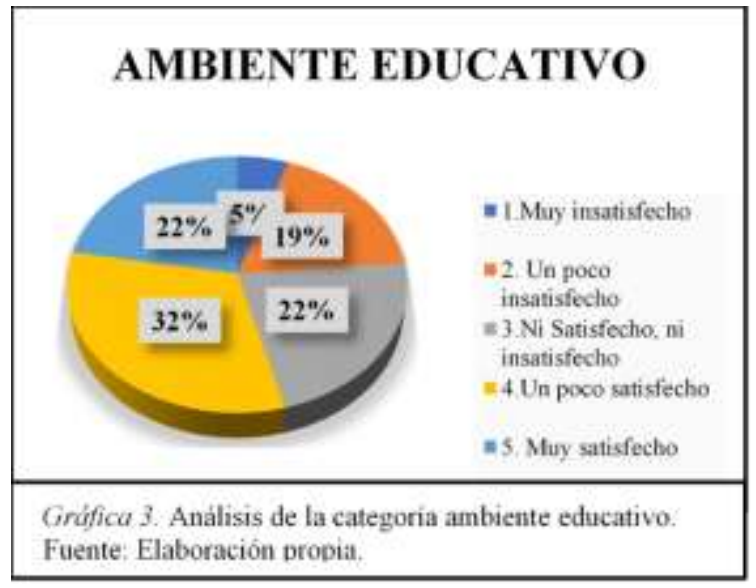

En la dimensión de organización académica el 35\% de los estudiantes se encuentran un poco satisfechos con los servicios que ofrece dicha categoría y el $7 \%$ se encuentran muy insatisfechos los servicios que integran la categoría son: la cantidad de estudiantes por salón de clases y la manera de organizar el horario de clases. (Vea Gráfica 4)

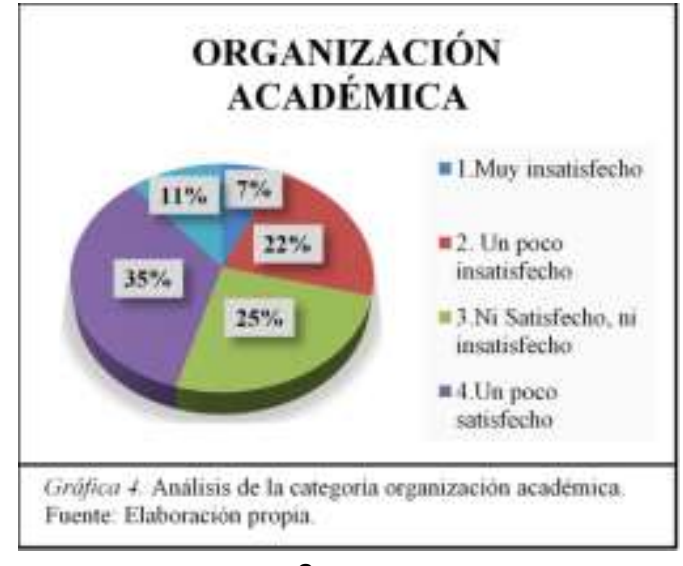


En la dimensión de bienestar estudiantil el 35\% de los estudiantes opinan que no se encuentran ni satisfechos ni insatisfechos y el 7\% se encuentran muy insatisfechos con la categoría. Los servicios que integran la categoría son: el respeto por las opiniones, sentimientos o preocupaciones, calidad de apoyo psicológico. El mostrar preocupación cuando un estudiante tiene algún problema. (Vea Gráfica 5)

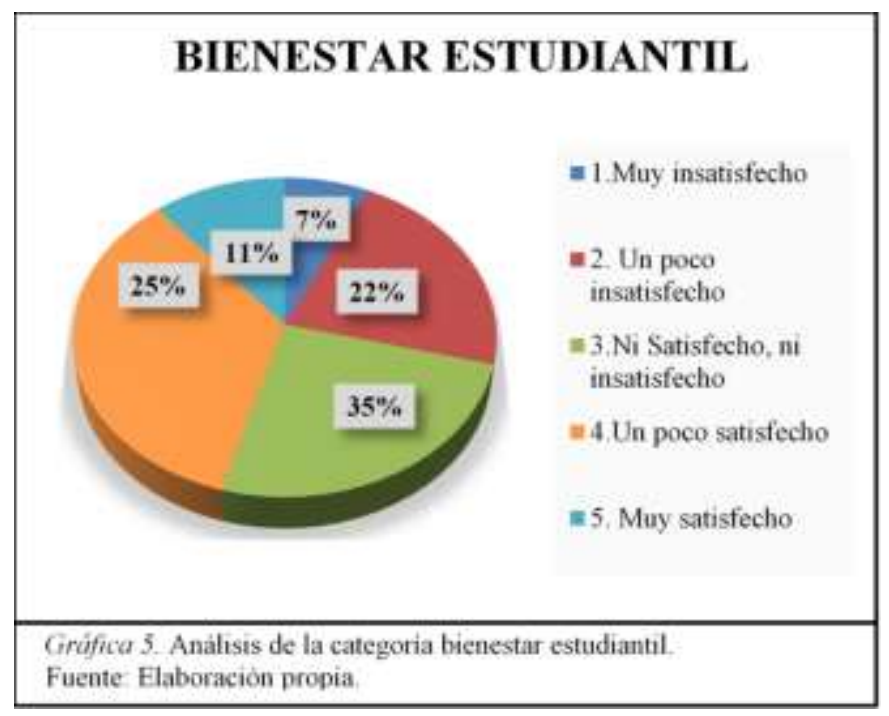

En la dimensión de calidad administrativa el 34\% de los estudiantes se encuentran un poco satisfechos con la disponibilidad y amabilidad del personal administrativo. Aunque en algunas ocasiones el trato no es el correcto. (Vea Gráfica 6).

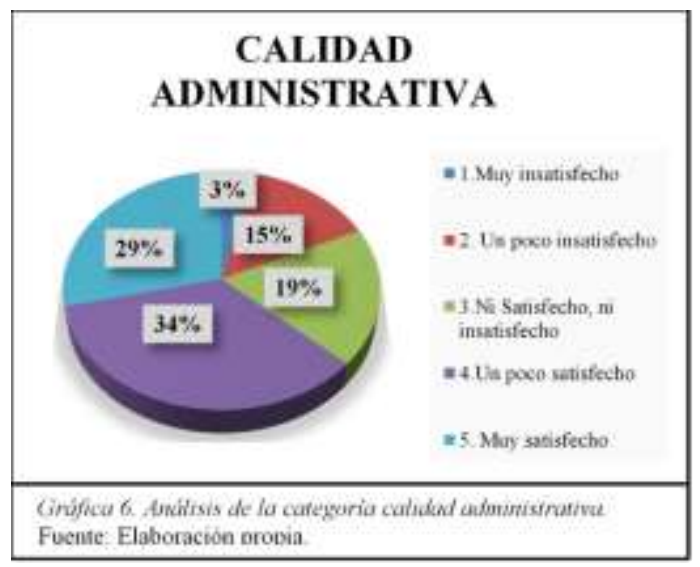


En la dimensión de calidad docente el $38 \%$ de los estudiantes se encuentran un poco satisfechos y solo el 5\% opina que se encuentran insatisfechos con la experiencia y la calidad de enseñanza del personal docente. Depende de qué tipo de profesor se le asigne pero que por lo general el maestro cumple el perfil. (Vea Gráfica 7)

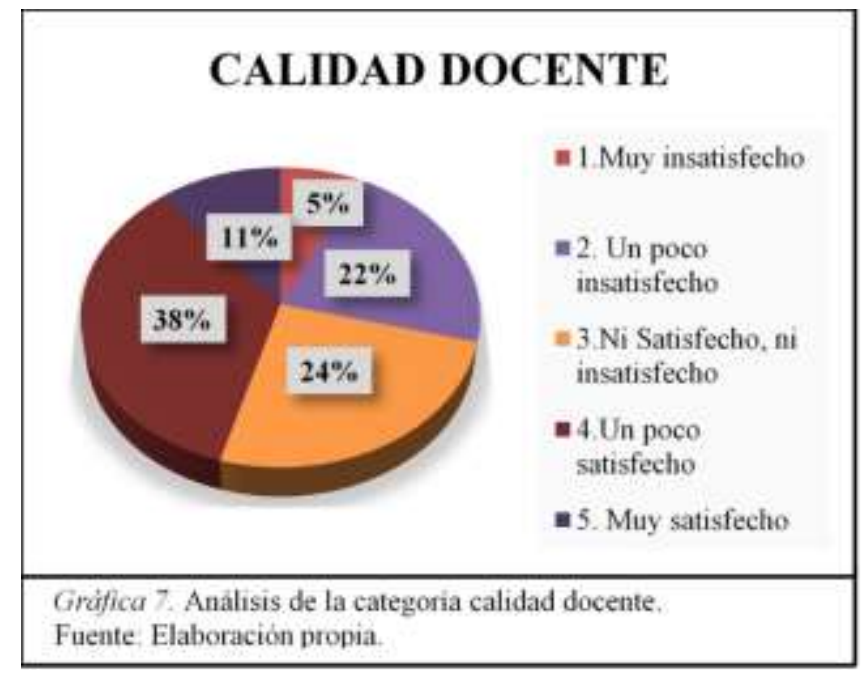

En la dimensión de infraestructura educativa el $36 \%$ de los estudiantes se encuentran un poco satisfechos con el mobiliario y la iluminación de los salones de clases y el 8\% opina lo contrario. (Vea Gráfica 8)

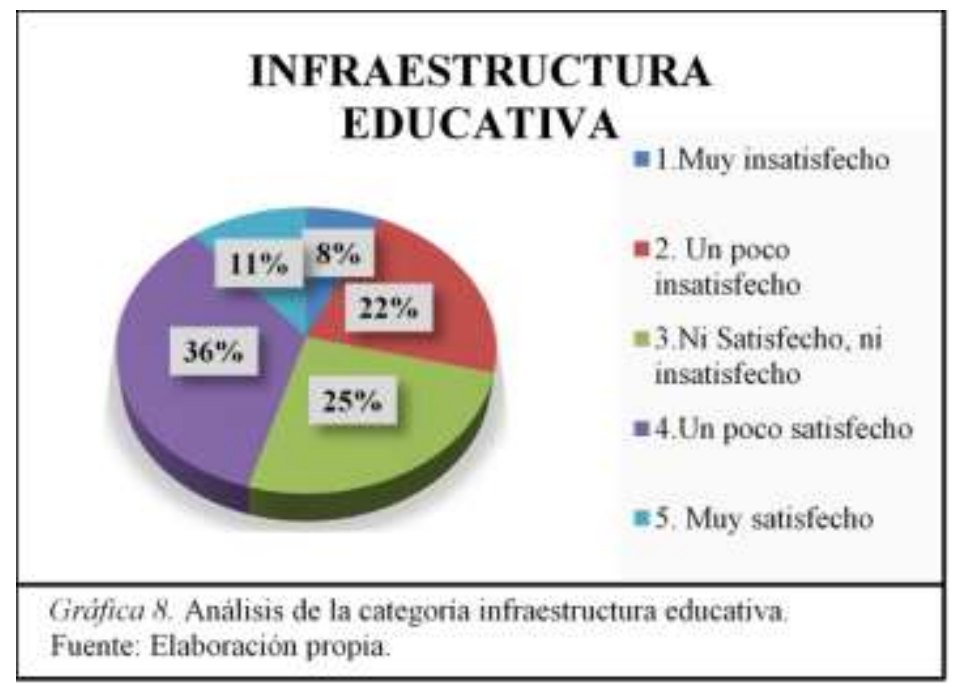


En la dimensión de recursos para la investigación el 30\% de los estudiantes se encuentran un poco satisfechos con la disponibilidad de los libros en la biblioteca tanto física como virtual y solo el 9\% opina lo contrario. (Vea Gráfica 9)

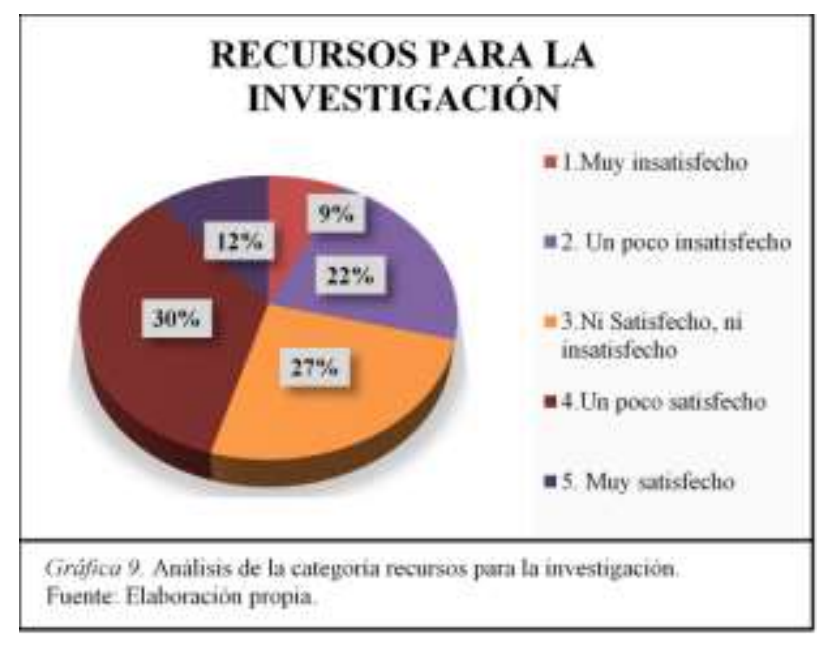

En la dimensión de servicios estudiantiles el $45 \%$ de los estudiantes se encuentran un poco insatisfechos con los servicios higiénicos y servicios de comida en la universidad, ya que no se ofrecen de manera correcta, los baños nunca tienen papel higiénico y a veces ni agua, los servicios de comida no se ofrecen ya que se encuentra cerrada la cafetería de la universidad. (Vea Gráfica 10)

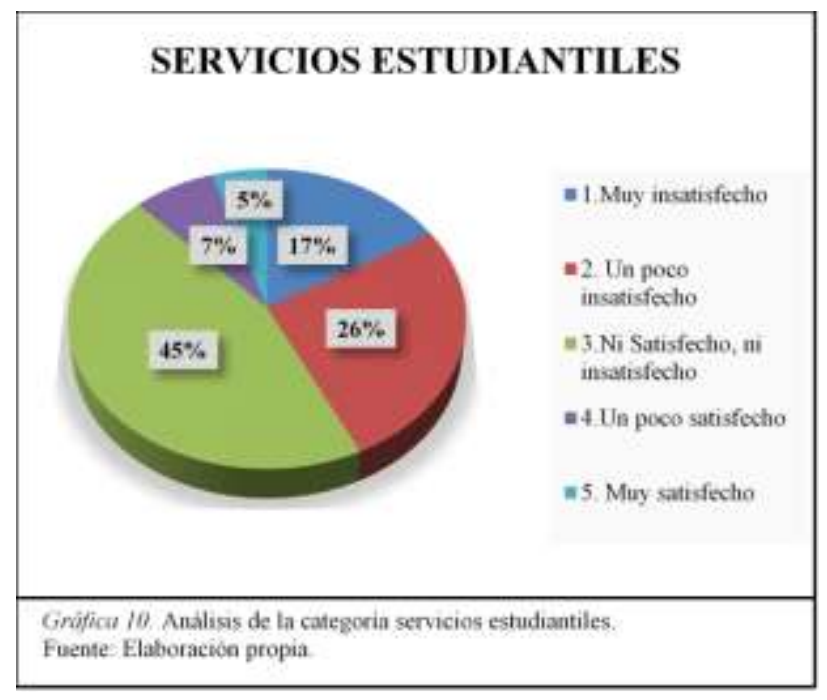


En la dimensión servicios informáticos el $29 \%$ de los estudiantes se encuentran un poco insatisfechos con la plataforma virtual en los servicios de reinscripción y velocidad del internet en la universidad, opinan que el internet solo se otorga a unos cuantos, y además no es gratuito, la plataforma de inscripción se satura mucho y luego no te ofrecen las materias que necesitas, mencionan que se debería resolver ese problema. (Vea Gráfica 11).

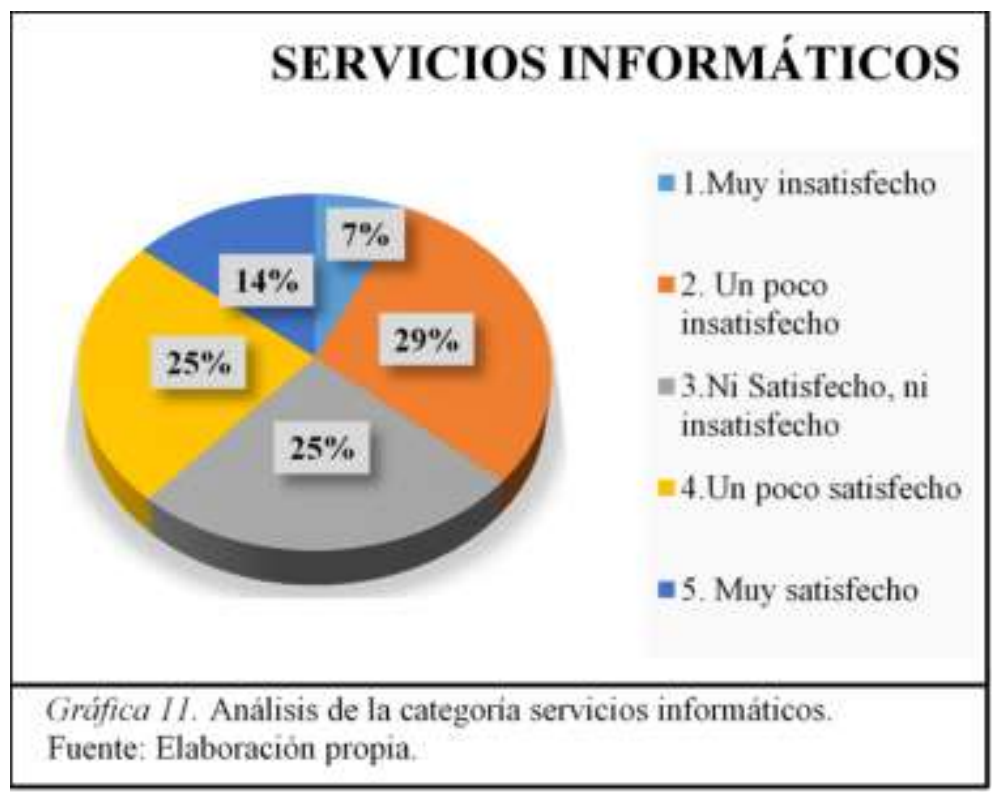

En la dimensión de vinculación con la sociedad el $45 \%$ de los estudiantes opina que no se encuentra ni satisfechos, ni insatisfechos con las oportunidades para conseguir empleo en la universidad. Cada quien busca empleo de acuerdo a sus perfiles.

\section{Discusión}

El cuestionario refleja que la mayoría de los estudiantes se encuentran un poco satisfechos con los servicios universitarios en general, aunque no garantiza que se estén ofreciendo del todo bien, ya que se pudo apreciar que algunos estudiantes opinaron que los servicios estudiantiles que se ofrecen no son de buena calidad, es por ello que se debe diseñar acciones para brindar un excelente servicio para impulsar el desempeño académico de estudiantes de posgrado que al estar en el PNPC demandan dedicación del tiempo completo a sus estudios. Las autoridades de la institución deben 
conocer la problemática para corregirla y así apoyar la correcta formación académica de los alumnos.

\section{Conclusiones}

Una dificultad en los esfuerzos de aumentar la calidad en los servicios a los estudiantes es el desconocimiento de cuáles son los factores importantes para los estudiantes por lo cual se dispuso a darlos a conocer a los estudiantes y que estos opinaran que tan satisfechos estaban con ellos.

Por lo cual se concluye que la satisfacción de los alumnos siempre debe ser muy importante en todas las instituciones a nivel superior, medio superior o básico, ya que como se ha mencionado afecta mucho a la correcta formación académica de los estudiantes, si se forman estudiantes poco contentos con su ambiente educativo, puede ocasionar que su rendimiento académico disminuya y por consiguiente sus calificaciones no serían buenas, como son estudiantes de posgrados con altas

exigencias académicas están presionados para cumplir con los requerimientos además de que cualquier error puede ocasionar que abandone sus estudios de posgrado por no respetar dichas exigencias y en consecuencia el nivel de eficiencia terminal sería menor.

\section{Bibliografía}

Aguerrondo, I. (2003). Formación docente desafíos de la política educativa. . Secretaría de Educación Pública.

Allen, M., Bourhis, J., Burrell, N., \& Mabry, E. (2002). Comparing student satisfaction with distance education to traditional classrooms in higher education: A meta-analysis. American Journal of Distance Education, 16(2), 83-97.

Bendermarcher, G. Egbrink, O. Wolfhagen, I. \& Dolmas, D. (2017) Desentrañar la cultura de calidad en la educación superior: una revisión realista. Educación más alta, 39-60.

Sverdlick, i. (2012). Buscando a la calidad educativa. Buenos Aires: noveduc (novedades educativas).

Bustos, T., Salazar, A., \& Bermúdez, J. (2012). Percepción de directivos, docentes y estudiantes, en torno a la implementación de un sistema de aseguramiento de la calidad en las instituciones educativas. Tesis de licenciatura. Chía: Universidad de la Sabana., p.14. 
Cadena Badilla, M., Mejias Acosta, A., Vega Robles, A., \& Vásquez Quiroga , J. (2015). La satisfacción estudiantil universitaria: análisis estratégico a partir del análisis de factores. Revista de la Facultad de Ingeniería Industrial, 18(1): 9-28.

Capelleras, J., \& Veciana, J. (2004). Calidad de servicio en la enseñanza universitaria: desarrollo y validación de una escala de medida. Revista Europea de Dirección y Economía de la Empresa, 13(4), 55-72. .

Conacyt. (2019). Conacyt. Recuperado el 11 de OCTUBRE de 2019, de https://www.conacyt.gob.mx/index.php/becas-y-posgrados/programa-nacional-deposgrados-de-calidad

DACEA. (2019). Universidad Juarez Autonoma de Tabasco. Recuperado el 11 de Octubre de 2019, de http://www.ujat.mx/

Eom, S., Wen, H., \& Ashill, N. (2006). The determinants of students' perceived learning outcomes and satisfaction in university online education: An empirical investigation. . Decision Sciences Journal of Innovative Education, 4(2), 215-235. .

Gento Palacios, S., \& Vivas García, M. ( 2003). El SEUE: un instrumento para conocer la satisfacción de los estudiantes universitarios con su educación. Acción Pedagógica, 1627.

González Peiteado, M., Pino Juste, M., \& Penado Abilleira, M. (2017). Estudio de la satisfacción percibida por los estudiantes de la UNED con su vida universitaria. Revista Iberoamericana de Educacion a Distancia, 243-260.

Groenewegen, P., \& Hutten, J. (1991). Workload and job satisfaction among general practitioners: A review of the literature. . Social Science \& Medicine., 32(10), 1111-1119.

Jacqueline, Alex, D., \& Barry, B. (2006). La medición de la satisfacción del estudiante en una Universidad de UK. Emerald Insight, 251-267.

Lomas, L., MacGregor, J., \& Hill, Y. (2003). Students' perceptions of quality in higher education. Quality Assurance in Education, 11(1), 15-20.

Medrano, L. A., \& Perez, E. (2010). Adaptación de la Escala de Satisfacción Académica a la Población. SUMMA Psicológica UST, 5-14.

Rebollo Meza, J. (2018-2019). $4^{\circ}$ Informe de Actividades DACEA.

Salvador, C. (2005). La percepción del cliente de los elementos determinantes de la calidad del servicio universitario: características del servicio y habilidades profesionales. Papeles del Psicólogo, 26(90), 1-9. 
Sánchez López, M. C., García Sánchez , F. A., \& Martínez Segura, M. J. (2012). Aproximación A La Valoración Que El Alumnado Hace De Recursos Online Utilizados Para La Docencia Universitaria. Revista de Medios y Educación, 35-45.

Sanjuán Gómez, G., Gómez Martínez, M., Rabell, P., Arcia, L., \& Morales Velázquez, I. (2011). Resultados preliminares del grado de satisfacción con el empleo del aula virtual de la Facultad de Ciencias Médicas General Calixto García. Revista Habanera de Ciencias Médicas., 10(1), 114-125. .

Senlle, A., \& Gutiérrez, N. (2005). CALIDAD EN LOS SERVICIOS EDUCATIVOS. España: Díaz de Santos.

Tam, M. (2002). Measuring the effect of higher education on university students. Quality Assurance in Education., 10(4), 223-228.

Zambrano, J. (2019). Cuestionario para medir la importancia y satisfacción de los servicios universitarios desde la perspectiva estudiantil. Revista Ciencia UNEMI, 35-45.

Zambrano, R. (2016). Factores predictores de la satisfacción de estudiantes de cursos virtuales. RIED. Revista Iberoamericana de Educación a Distancia., 19(2), 217-235. 


\section{Directorio Institucional}

Dr. Enrique Fernando Velázquez Contreras

Rector

Dra. Arminda Guadalupe García de León Peñuñuri

Secretario General Académico

Dra. Rosa Mará Montesinos Cisneros

Secretaria General Administrativa

Dra. Ramón Enrique Robles Zepeda

Director de Investigación y Posgrado

Dr. Rodolfo Basurto Álvarez

Director de Vinculación y Difusión

Dra. Adriana Leticia Navarro Verdugo

Vicerrectora de la Unidad Regional Sur

Dr. Ernesto Clark Valenzuela

Director de la División de Ciencias Económicas y Sociales

Dr. Francisco Espinoza Morales

Secretario de la División de Ciencias Económico y Sociales

Dra. Leticia maría González Velásquez

Jefe del Departamento de Ciencias Económico Administrativas

Dra. Lidia Amalia Zallas Esquer

Jefe de Departamento de Ciencias Sociales 


\section{Comité Directivo}

\section{Editor Responsable}

Dr. Francisco Espinoza Morales

Directora

Dra. Leticia María González Velásquez

Subdirector

Dr. Javier Carreón Guillen

Editor Científico

Dr. Cruz García Lirios

Master Gráfico

M.T.I. Francisco Alan Espinoza Zallas

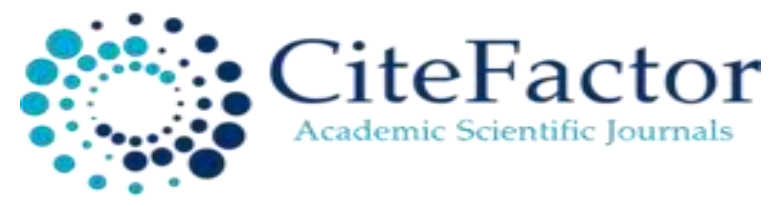

"Academic Research Journal Withoutborders

Nos complace anunciar que su diario, "Academic Research Journal Withoutborders" (ISSN/EISSN 20078870) fue evaluado positivamente en la indexación Citefactor, ahora la página de la revista está disponible en línea, en caso de cualquier problema / actualización que pueda enviarnos por correo a support@citefactor.org. 


\section{Comité editorial}

Dra. Angélica María Rascón Larios

Universidad de Sonora. México

Dra. María del Rosario Molina González

Universidad de Sonora

Dra. Francisca Elena Rochin Wong

Universidad de Sonora. México

Dra. Lidia Amalia Zallas Esquer

Universidad de Sonora. México

Dra. Beatriz Llamas Arechiga

Universidad de Sonora. México

Dr. Rogelio Barba Álvarez

Universidad de Guadalajara. México

Dra. Rosa María Rincón Ornelas

Universidad de Sonora. México

Dr. Juan Flores Preciado

Universidad de Colima. México

Dr. Amado Olivares Leal. Universidad de Sonora

Universidad de Sonora. México

Dr. Guillermo Velázquez Valadez.

Instituto Politécnico Nacional (IPN) México 
Dr. Hugo Nefstalí Padilla Torres.

Universidad Estatal de Sonora. México

Dr. Luis Ramón Moreno Moreno.

Universidad Autónoma de Baja California. México

Dr. Miguel Ángel Vázquez Ruiz.

Universidad de Sonora. México

Dra. Lorena Vélez García.

Universidad Autónoma de Baja California. México

Dra. Pabla Peralta Miranda.

Universidad Simón Bolívar, Barranquilla, Colombia

Mtro. Roberto Espíritu Olmos

Universidad de Colima (FCA Tecomán) Colima

Dr. Héctor Priego Huertas.

Universidad de Colima (FCA Tecomán) Colima

Mtra. María Guadalupe Alvarado Ibarra.

Universidad de Sonora. México.

MSc. Celso Germán Sánchez Zayas

Universidad de Camagüey, Ignacio Agramonte Loynaz, Cuba

Dra. María Luisa Quintero Soto

Universidad Autónoma del Estado de México

Dr. Eyder Bolivar Mojica

Universidad Católica, Luis Amigó, Medellin, Colombia

Revisores de Textos en Inglés

Mtro. Renato Encinas

Mtra. Cecilia Guadalupe Martínez Solano 


\title{
Comité científico
}

\author{
Dr. Rosendo Martínez Jiménez. Universidad Autónoma Benito Juárez de Oaxaca. \\ Dr. Hugo Neftalí Padilla. Universidad Estatal de Sonora \\ Dra. María Teresa Gaxiola Sánchez. Universidad de Sonora. \\ Dr. José Cesar Kaplan. Universidad Estatal de Sonora. \\ Dr. Alfredo Islas Rodríguez. Universidad de Sonora
}

Frecuencia de publicación: semestral / 2 números por año.

Revista de Investigación Académica sin Frontera (RIASF) con (ISSN: 2007-8870) es un interlocutor internacional de acceso abierto revisado diario en línea en el ámbito del de las Ciencias Económicas Administrativas y Sociales. Su objetivo principal es dar a los trabajos de investigación de calidad. Cubre todas las sub-campos de los campos anteriormente mencionados. Proporciona la plataforma a académicos, estudiantes y profesionales. Sólo pública trabajos de investigación y artículos de revisión inicial. Documento presentado debe cumplir con algunos criterios como, debe ser original, inédita y no estén sometidos a ninguna otra revista. RIASF es una revista arbitrada / Revisión por pares International. Publicamos documentos sobre una variedad de temas, contextos y estrategias de análisis que examinan la relación entre la rápida evolución para la Sociedad y la tecnología del conocimiento.

REVISTA DE INVESTIGACIÓN ACADÉMICA SIN FRONTERA, Año 13, No. 32, Enero-junio 2020, es una publicación semestral de investigación científica, editada por la Universidad de Sonora, a través de las División de Ciencias Económicas y Sociales, de la Unidad Regional Sur, Blvd. Lázaro Cárdenas No. 100, Col. Francisco Villa, Navojoa, Sonora, Sonora, México, C.P. 85880. Tel. (642) 425- 99-54. http://www.revistainvestigacionacademicasinfrontera.com/, revistaacademicasinfrontera@ unison.mx.

Editor responsable: Francisco Espinoza Morales. Reserva de Derechos al Uso Exclusivo: 04-2013121811323700-203 e ISSN: 2007-8870, ambos otorgados por el Instituto Nacional de Derecho de Autor. Inscrita en el Directorio de LATINDEX, con Núm. De folio 20014, folio único 14590. Indexado a CiteFactor Academic Scientific Journal. Responsable de la última actualización de este Número, Unidad Informática de la Universidad de Sonora, fecha de la última modificación, 30 de junio 2020. Las opiniones expresadas por los autores no necesariamente reflejan la postura del editor de la publicación. Se autoriza la reproducción total o parcial de los contenidos e imágenes en la presente publicación siempre y cuando se cuente con la autorización del editor y se cite plenamente la fuente. 